\title{
ALCUNE RIFlessioni A PROPOSITO di UNA RECENTISSIMA GRAMMATICA DELLA LINGUA ITALIANA
}

1. L'Italia, che neppure nel passato mancava di grammatiche di indirizzo normativo e descrittivo, si è arricchita negli ultimi anni di una serie di opere glottodidattiche dedicate all'italiano. Una delle ultime è il poderoso volume La lingua e i testi, Grammatica della lingua italiana di P. Agazzi, A. Fallica e A. Menegoi, edito da Minerva Italica, Bergamo, 1988. Il libro non è soltanto una grammatica in senso usuale: infatti, vi si trattano le nozioni fondamentali della teoria linguistica attuale (comunicazione, segno linguistico, codice, funzioni della lingua, metafora e metonomia, denotazione e connotazione, fattori della comunicazione), della teoria del testo (con alcuni campioni di testi di vario genere), in seguito l'origine e lo sviluppo della lingua italiana (dall'Indovinello veronese ai nostri giorni), i dialetti e le comunità alloglotte, e nell'ultima delle quattro appendici si danno gli elementi della composizione scritta. Il volume include dunque in notevole misura la sociolinguistica, la pragmatica, la comunicazione e la teoria dell'informazione (invece di parlante o locutore e collocutore si usano conseguentemente i termini emittente e destinatario), la storia della lingua, la cultura del parlato e dello scritto. Si cerca insomma di avvicinare il Linguaggio all'alunno e di sviluppare in lui non solo la competenza grammaticale ma anche quella comunicativa, attiva e passiva.

2. Sebbene gli autori affermino di partire dalle teorie linguistiche correnti senza novità teorico-metodologiche (Prefazione, p. 6), alcune innovazioni importanti ci sono. A detta degli autori stessi, esse si trovano in tre domini: classi di parole, frasi nucleari e categorie del verbo (loco cit.); ma va sottolineato che anche l'impostazione stessa dell'opera, con i suoi orizzonti larghi, è un'innovazione rispetto alle grammatiche tradizionali; e ci sono anche diversi punti più speciali (v. av.). Si sente che gli autori hanno cercato di adottare una posizione di mezzo tra la grammatica di stampo antico ( dura disciplina che si imponeva con l'autorità di una legge di natura», p. 5) e il principio della libertà linguistica degli anni '60 (simboleggiato dallo slogan «val più la pratica che la grammatica», ib), sottomettendo gli eccessi di quest'ultimo atteggiamento ad una revisione e un ripensamento (ib.). Ne risultano alcuni tentativi di classificazione, sistematizzazione ed interpretazione in parte esatti, in parti discutibili (in quanto troppo semplificati), in parte finalmente inadeguati o francamente errati. Non potendo tuttavia presentare in questa sede una vera e propria recensione dell'opera, ci limitiamo a discutere alcune questioni che ci sembrano di particolare importanza, e precisamente: certe nozioni di teoria linguistica e linguistica generale $(\S 3)$, determinati problemi dell'analisi morfematica $(\S 4)$; lo status dell'elativo $(\S 5)$ e quello degli avverbi $(\S 6)$, alcune questioni di sintassi della frase $(\S 7)$ e infine determinati problemi della formazione delle parole $(\S 8)$. 


\section{Su certe nozioni di teoria linguistica e linguistica generale}

3.1 Alla p. 38, nel capitolo sull'arbitrarietà e convenzionalità del segno linguistico, si legge quanto segue:

«Non c'è niente, infatti, di intrinsecamente particolare nel referente che giustifichi la scelta di un certo significante piuttosto che di un altro.

Sono stati gli uomini appartenenti allo stesso gruppo sociale che, a un certo momento, si sono messi d'accordo fra di loro e, in virtù di una convenzione, hanno dato quel determinato nome che è stato utilizzato in quella forma da tutti i membri della stessa comunità.».

La prima parte del passo commenta l'arbitrarietà del segno linguistico ed è esatta e pacifica; la seconda, invece, suggerisce qualcosa di inaccettabile. Infatti, essa sembra ritornare all'antica discussione sull'attribuzione del significato physei o nomo (thesei), e precisamente a questa seconda tesi, lasciando intendere che gli uomini ad un certo momento si sono messi d'accordo di chiamare un dato oggetto con un determinato nome. Si sa da tempo che il segno linguistico (più precisamente, il rapporto tra significante e significato) è, sì, arbitrario, ma quest'arbitrarietà non implica alcuna decisione cosciente, presa ad una data tappa della storia, bensí si inquadra nella trasmissione storica del linguaggio. A quanto possiamo risalire nel tempo, ogni generazione eredita la lingua già fatta dalla generazione precedente. Classiche in questo senso sono le parole di F. de Saussure, «padre» incontestato del concetto dell'arbitrarietà del segno linguistico; ad es. (Saussure 1965):

«Il [ = le mot arbitraire] ne doit pas donner l'idée que le signifiant dépend du libre choix du sujet parlant [...]» p. 101)

«La langue ne peut donc plus être assimilée à un contrat pur et simple [...] si l'on veut démontrer que la loi admise dans une collectivité est une chose que l'on subit, et non une règle librement consentie, c'est bien la langue qui en offre la preuve la plus éclatante.» (p. 104)

e soprattutto:

«A n'importe quelle époque et si haut que nous remontions, la langue apparaît toujours comme un héritage de l'époque précédente. L'acte par lequel, à un moment donné, les noms seraient distribués aux choses, par lequel un contrat serait passé entre les concepts et les images acoustiques - cet acte, nous pouvons le concevoir, mais il n'a jamais été constaté.» (p. 105)

Da Saussure fino ad oggi si è ripetuto un'infinità di volte che la lingua è un fenomeno sociale, a cui l'individuo non può cambiare praticamente nulla; fenomeno ereditato da generazioni anteriori ed elaborato nel corso della storia, non creato ad una sua tappa per decisione cosciente. Ciò vale beninteso anche per il rapporto tra significante e significato. 
3.2 Alla p. 375 , nell'ambito del capitolo sul soggetto, si dice che il soggetto è costituito per lo più da un nome o da un pronome, ma che «qualsiasi elemento può fungere da soggetto (persino un articolo o un funzionale in frasi del tipo: il è un art $i$ colo determinativo; sebbene è una congiunzione) [...]». L'affermazione (che si leggeva anche in talune grammatiche di vecchio tipo) non distingue il piano linguistico da quello metalinguistico (eppure, a p. 103 la funzione metalinguistica è introdotta e commentata). Sul piano metalinguistico, infatti, q u al si a si parola può essere soggetto, anche una forma verbale personale ( = tradizionalmente: esplicita): «porterebbe è una forma verbale italiana» ecc. Ma in tutti questi casi si tratta di parole ci tat e, situate dunque su un altro piano, quello metalinguistico. Sul piano linguistico il, sebbene, porterebbe ecc. non possono mai funzionare da soggetto.

\section{Alcuni problemi dell'analisi morfematica}

4.1 Gli autori introducono il concetto di morfema nella solita accezione e ne distinguono alcune categorie (p. $199 \mathrm{sgg}$.). Nell'analisi delle forme verbali lodasti, tememmo, dormirono, condotta alla p. 250, si isola tuttavia, tra il morfema lessicale e quello grammaticale, una vocale definita caratteristica, così presentata:

$$
\text { lod-a -sti tem- }[-m \text {-mo dorm- } i-\text {-rono }
$$

Secondo questa vocale caratteristica (a cui si accenna già nella nota 2 della p. 201) si distinguono in italiano tre coniugazioni: gli infiniti della prima coniugazione escono in -are, della seconda in -ere e della terza in -ire.

Nello stesso passo i segmenti -v-(di lodavamo) e -r- (di temerà, dormiresti) sono definiti morfemi formativi.

Diverse obiezioni si possono fare.

1) Dapprima una questione terminologica. Secondo il nostro parere, il termine coniugazione andrebbe riservato al sistema della flessione verbale in genere; in altri termini, in una lingua non ci possono essere più coniugazioni ma una sola (se, cioè, le categorie verbali si esprimono mediante desinenze, alternanze ecc.). Per quelle che la grammatica tradizionale denomina coniugazioni, preferiamo il termine classi, sicché ci sarebbero in italiano tre classi :-are, -ere, -ire (ma v. un po' av.), In tal modo si evita l'ambiguità del termine coniugazione la quale, portata all'estremo, permetterebbe di dire ad esempio che in italiano la coniugazione [nel nostro senso] distingue tre coniugazioni [nel senso degli autori].

2) A dir vero, la divisione nelle tre classi citate non è sufficiente, ossia la divisione basata unicamente sulla vocale «caratteristica» non basta. Infatti, all'interno della I classe cantare, andare, dare e fare ad es., non possono far parte dello stesso tipo, perché si distinguono in determinate forme; la III classe si divide almeno in tre sottoclassi (sentire senza -isc-, finire con -isc-, venire senza -isc-ma con l'alternanza 
e con il part. in -uto), e una varietà ancor maggiore si riscontra nella II classe (a cominciare dall'infinito: vedére : véndere).

3) La vocale «caratteristica» è un po' «campata nell' aria», dato che il suo status morfematico non è definito. Giacché si adotta l'analisi in morfemi, ogni segmento di una forma verbale deve far parte di un morfema o essere un morfema a sé stante. Quale di queste due possibilità vale a proposito della vocale «caratteristica»? Se è morfema a sé, bisognerebbe specificare che contenuto gli corrisponde; se non è un morfema autonomo, di quale dei due altri morfemi fa parte: di quello lessicale o di quello grammaticale? Nella nostra grammatica non apprendiamo niente in merito.

4) Infine, non ci sembra adatto denominare i segmenti - $v$ - e - $r$ - morfemi formativi, perché il termine può creare confusione con il dominio della formazione delle parole (infatti, così lo stesso termine viene usato alle pp. 204-205). Sarebbe certamente preferibile definire questi segmenti ad esempio come morfemi temporali e/o modali.

4.2 Anche in uno degli esercizi alla p. 205 si pone il problema dello status dei segmenti ottenuti dall'analisi delle parole. L'alunno deve distinguere i morfemi lessicali, grammaticali e formativi in alcune serie di esempi, tra cui anche la serie affet$t i$, affettivo, affettuose, affettuosamente. Nessuna difficoltà quanto alle prime due parole; ma come analizzare il segmento - $u$-nelle altre due? Nessuna istruzione a proposito; e anche più tardi, tra gli esempi per il suffisso -oso (pp. 537-538), non si dà alcun esempio in -uoso. Teoricamente ci sono tre possibilità: a) isolare - $u$-come un morfema a sé (afett-u-oso), dunque farne una specie di vocale «caratteristica» di questo tipo di derivati (soluzione da scartare essendo $-u$ - privo di significato); b) interpretare affettu- come allomorfo del morfema lessicale (soluzione forse preferibile dal punto di vista diacronico); c) interpretare -uoso come allomorfo del suffisso -oso (soluzione probabilmente più accettabile dal punto di vista sincronico). Come si regolerà l'alunno in tali casi? Non sarebbe stato preferibile dare ad es. la serie rispetto, rispettoso, rispettosamente?

4.3 La tabella in fondo alla p. 269 riassume le desinenze del nome dividendole in quattro gruppi. Il primo gruppo è quello dei nomi poema e rosa $(-a,-i,-e)$, il secondo quello dei nomi lupo e mano $(-o,-i)$, il terzo gruppo racchiude i nomi tipo monte e trave $(-e,-i)$, mentre il quarto presenta «diverse desinenze», con l'indicazione «Non variano al plurale» e gli esempi auto, crisi, virtù, film, sport... Poiché la -o di auto, la - $i$ di crisi e la -ù di virtù non si possono in alcun modo isolare come desinenze (e in film, sport ecc. non ci sono affatto vocali finali!), nel quarto gruppo non si può parlare di «diverse desinenze» ma tutt'al più di una desinenza al grado zero. Ma uno zero ha senso e giustificazione soltanto se si oppone ad un altro elemento che non sia zero; e siccome qui anche il plurale dovrebbe presentare la stessa desinenza al grado zero, è senz'altro più semplice e più aderente alla realtà concludere che nei nomi auto, crisi, sport ecc. non ci sono desinenze. 
4.4 Per alcuni problemi dell'analisi morfematica nei suffissi rimandiamo al $\S 8$.

\section{Lo status dell'elativo}

Anche la grammatica dei nostri tre autori appartiene a quella schiera di grammatiche italiane che, trattando dei gradi dell'aggettivo, distinguono il superlativo relativo dal superlativo assoluto, il che significa che quest'ultimo viene incluso nella comparazione. Si ha il superlativo relativo «quando si mantiene il confronto tra un nome e il gruppo di cui il nome fa parte» (un esempio: Giove è il più grande dei (fra i) pianeti), mentre abbiamo il superlativo assoluto «quando si elimina il termine di confronto» (esempi: Marta è una bellissima bambina, questo monte è altissimo, Marta è molto bella) (p. 332). Sull'assurdità del concetto di superlativo assoluto e sulla necessità di escluderlo dalla comparazione abbiamo scritto a diverse riprese (Tekavčić 1968a, 1968b, $1980 \S 487,1982$ ) sicché non intendiamo ripeterci in questa sede. Ricordiamo soltanto che più di trent'anni fa $\mathrm{B}$. Pottier constatava che il concetto di superlativo assoluto è privo di senso perché la nozione stessa di 'superlativo' implica un riferimento [dunque, un confronto] (Pottier 1958, pp. 111-112). Le due forme sono distinte e non sono intercambiabili; si confronti:

ho visto una bellissima ragazza

*ho visto una la più bella ragazza

Laura è la più bella delle alunne

* Laura è bellissima delle alunne

Le due funzioni sono distinte sia per forma che per contenuto, e ciò vale anche per le forme sintetiche ereditate: massimo e minimo sono normalmente superlativi relativi, non assoluti, mentre ottimo e pessimo sono superlativi assoluti, non relativi (il massimo di tutti — *è massimo; è ottimo — *l'ottimo di tutti ecc.).

Nell'italiano attuale, dunque, non c'è nessuna ragione plausibile per includere il superlativo assoluto nella comparazione; anzi, procedendo cosi, si creano inutili difficoltà ai discenti alloglotti, le cui lingue materne non distinguono due superlativi. Proponiamo perciò di separare la trattazione di bellissimo da quella di il più bello; nel contempo, riteniamo del tutto giustificato sostituire il termine superlativo relativo con superlativo (senza precisazioni) e sostituire l'assurdo superlativo assoluto con elativo. D'ora in poi ci serviremo di questi termini.

5.2 Un'evidente concessione alla grammatica più tradizionale - il che sorprende in un manuale peraltro impostato così modernamente! - è l'inclusione nella comparazione anche delle forme anteriore, posteriore ecc.; primo, ultimo, infimo, intimo, estremo (p. 334). Anzi, persino in queste forme (o meglio: parole, poiché si tratta di unità lessicalizzate) si cerca di distinguere il superlativo dall'elativo (superlativo: l'intimo; elativo: intimo). Di conseguenza, dovrebbero essere possibili frasi come *Pietro è davvero intimo (cfr. Pietro è davvero gentilissimo) e *Pietro è 
l'intimo di tutti i miei amici (cfr. Pietro è il più gentile di tutti i miei amici). Eppure, è ovvio che questi esempi sono inaccettabili.

5.3 Più grave ancora è l'inadeguatezza della presentazione della comparazione degli avverbi: nel superlativo degli avverbi non si fa la distinzione tra superlativo ed elativo ma si dà un solo superlativo (senza precisazione), e precisamente solt an to le forme benissimo o ottimamente, malissimo o pessimamente, massimamente o sommamente, moltissimo, pochissimo o minimamente (p. 350). Tutte queste forme sono unicamente elativi e pertanto inutilizzabili nella comparazione: prova ne sia l'agrammaticalità di una frase come *Pietro ha giocato benissimo (o ottimamente) di tutti gli alunni. Gli autori non dicono una sola parola su come bisogna esprimere il superlativo degli avverbi (superlativo nel nostro senso). È vero che l'italiano non conosce un superlativo degli avverbi come lo possiede ad esempio il francese (le mieux, le plus gentiment ecc.), così che il corrispondente contenuto va espresso col comparativo precisato da un complemento adatto (meglio di tutti, meglio che mai) o mediante una perifrasi (nel miglior modo possibile, nel migliore dei modi ecc.). $\mathrm{E}$ questa una specialità della morfosintassi italiana, che non va trascurata nemmeno nella più sommaria presentazione della comparazione degli avverbi.

\section{Lo status della classe degli avverbi}

6.1 L'eterogeneità di questa infelice classe di parole è stata avvertita da tempo: B. Pottier, nella pubblicazione già citata, rinuncia al termine 'avverbio' (adverbe) per via dell'abuso che se n'è fatto (Pottier 1958, p. 96); più tardi, D. Crystal parla a proposito degli avverbi di una dustbin class 'classe - pattumiera' e aggiunge l'atteggiamento tradizionale: «se non si sa come definire una parola, la si chiami avverbio» (Crystal 1971, p. 75), e il Francese Jack Feuillet definisce a due riprese l'avverbio come una classe «fourre-tout» $(1983$, p. $38 ; 1987$, p. 20$)$. L'incomprensibile eterogeneità degli avverbi tradizionali fa sì che alcuni tra i migliori dizionari italiani (Devoto-Oli 1971, Zingarelli 1983) e alcuni dizionari bilingui (Deanović-Jernej 1980) definiscano come avverbio perfino la parola cioè! Purtroppo anche i nostri autori dividono gli avverbi in cinque tipi: 1 . di luogo, 2. di tempo, 3. di modo, 4. di quantità, 5. affermativi, negativi e dubitativi (p. $342 \mathrm{sgg}$.). Ma fra gli avverbi si trova incluso anche ecco ( A metà fra gli avverbi di luogo e gli avverbi di tempo», p. 345, scheda num. 17), nonché - cosa ancor più grave - le parole sì e no (p. 348). Eppure, sempre alla p. 348, si dice che sì e no sostituiscono un'intera frase! Di conseguenza, sì e no funzionano come sostituenti di frasi o sostituenti profrastici; allora, come possono nel contempo essere avverbi? Le due funzioni ovviamente si escludono. (Non si obietti che in esempi quali Come ha suonato Mario? - Bene l'avverbio sostituisce una frase: non la sostituisce ma rappresenta la risposta ellit t ic a ad una domanda nucleare; il sì, al contrario, sostituisce tutta una frase costituita da una domanda connessionale.) Una ventina di pagine dopo (p. 368), tuttavia, la parola ecco riappare, questa volta fra le - interiezioni! Ad illustrare questo secondo uso si dà un lungo esempio che comincia così: Ecco, vede signor commissario, io ero alla finestra [...]. Secondo noi, ecco non è né avverbio né interiezione: infatti, esso non puó 
determinare il verbo o l'aggettivo (il che è la funzione dell'avverbio) né può equivalere ad un intero enunciato (funzione dell'interiezione). In compenso, ecco può reggere una frase (ecco che viene il direttore, ecco come bisogna fare ecc.; $\mathrm{cfr}$. vedo che viene il direttore. ti dico io come bisogna fare) o un elemento nominale (ecco Mario, eccolo; cfr. vedo Mario, it. ant. vedolo): dunque si comporta sintatticamente come un verbo, sicché non ha avuto torto R. A. Hall jr. (1952-53) di definire ecco come una specie di verbo, formalmente ridotto al massimo; e cfr. i corrispondenti francesi voici, voilà, i quali contengono una forma verbale fossilizzata.

\subsection{A proposito della comparazione degli avverbi v. sopra, $\S 5.3$.}

\section{Alcuni problemi di sintassi della frase}

I nostri autori partono dall'affermazione del tutto esatta che le subordinatecomplemento (che sono il terzo gruppo, dopo le relative $/ 1^{\circ}$ gruppo/ e le soggettive ed oggettive $/ 2^{\circ}$ gruppo $/$ ) «sono da considerare [...] la trasformazione di complementi della frase semplice» (p. 485) e cercano pertanto di mettere in risalto le corrispondenze tra subordinate e complementi (ib.). Al termine, tuttavia, ammettono di dover rinunciare ad una trattazione esauriente e di aver fornito soltanto determinati tipi di frasi (p. 493); perciò, come in ogni classificazione linguistica, rimangono dei «resti», qui definiti «altri tipi di subordinate» (pp. 493-495). Sono frasi alle quali non corrisponde un complemento. Senonché, tra di esse ci sono anche le frasi avversative (con mentre e invece di) e le frasi aggiuntive (con oltre a/oltre che) (p. 494). Anche nella grande tabella riassuntiva (pp. 496-500) questi due tipi di frasi sono privi di complementi corrispondenti. Eppure, questi complementi esistono, ad esempio: invece del vino bevo solo succhi di frutta, oltre alla casa Pietro possiede anche una bella villa ecc. Non risulta dunque perché i due tipi citati siano sprovvisti di equivalenti nella categoria dei complementi.

7.2 Pur con le riserve formulate a p. 493 (a cui abbiamo accennato poco fa), gli autori hanno dato un vasto campionario delle frasi subordinate italiane. Uno dei tipi sono anche le frasi definite strumentali, con il verbo al gerundio (Riflettendo, si possono evitare errori) o all'infinito introdotto da con (Col riflettere, ecc.) (p. 496). A nostro parere, vi appartiene un terzo tipo, di cui la grammatica non dice nulla: quello con l'infinito introdotto da $a$, ad es.: hai fatto bene a prenotare $i$ posti a tempo. Queste frasi non sono né oggettive (risposta a: che cosa hai fatto?) né causali (perché lo hai fatto?) né modali (come hai fatto?) ecc., bensì specificano con quale mezzo o strumento (qui in senso figurativo) è stata compiuta l'azione espressa nella reggente (infatti, il verbo vi può essere anche al gerundio: hai fatto bene prenotando i posti a tempo).

7.3 La trattazione delle frasi interrogative (pp. 423, 506-507) non fa la distinzione dei due tipi di domande, da L. Tesnière denominati nucleari e connessionali (Tesnière 1959, pp. 192 sgg., 203 sgg.) e noti nella'linguistica anglosassone risp. come wh-questions e yes or no questions. Alle pp. 506-507 alle interrogative dei due 
tipi si aggiungono anche le domande alternative (chiamate dagli autori disgiuntive; es.: Dobbiamo restare o (dobbiamo) andarcene?) e quelle retoriche (es.: Forse che non siamo tutti mortali?). Qualche parola di commento sulle differenze tra questi tipi di frasi interrogative sarebbe certamente utile.

7.4 L'analisi delle frasi causali si svolge in due posti reciprocamente per così dire complementari, per cui l'esposizione perde la necessaria completezza. Alla p. 486 si illustra soltanto il tipo poiché $X \rightarrow$ allora $Y$ lla causa è nota, l'effetto è rematizzato come novum], mentre alla p. 497 (nella già citata tabella riassuntiva) si danno, è vero, tutte le congiunzioni causali, ma gli esempi illustrano soltanto il tipo contrario [Y perché $X$ : effetto noto, causa rematizzata]. Sarebbe senz'altro preferibile descrivere in ambedue i posti entrambi i tipi, e commentarne gli elementi identici e le differenze.

7.5 Gli autori includono tra le subordinate consecutive anche quelle che noi abbiamo definito frasi di [ $\alpha$ adeguatezza] (Tekavčić 1980, II, p. 455) (esempio degli autori: ero troppo affamato perché potessi (per poter) impegnarmi in una discussione, p. 489) nonché le frasi da noi denominate consecutive interrogative (Tekavčić, op. cit., pp. 455-456), ossia frasi motivanti una domanda o una presunzione (secondo Regula-Jernej 1975, p. 320) (esempio degli autori: cos'hai, che sei (da essere) cosi nervoso?, ib.). Che in questi due tipi di frasi ci sia un certo rapporto fondamentalmente consecutivo è indubbio, ma a nostro parere ciò non basta per considerarle consecutive (tant'è vero che, procedendo così, a rigor di termini anche le causali potrebbero essere annoverate tra le frasi consecutive). Le frasi consecutive interrogative non constatano un rapporto tra una causa effettiva ed una conseguenza altrettanto effettiva ma interrogano sulla causa (dunque, ignota) che può aver provocato la conseguenza, oppure ipotizzano una tale causa. Quanto all'altro tipo (frasi di $[\alpha$ adeguatezza]), neppure esse stabiliscono un rapporto effettivo tra causa e conseguenza, ma si limitano ad esprimere il grado della causa adeguato o meno (da qui il nostro termine) per determinare la relativa conseguenza. Ciò significa che nemmeno in queste frasi il rapporto tra causa e conseguenza è realizzato, per cui sul piano dell'espressione abbiamo il congiuntivo. Si confronti:

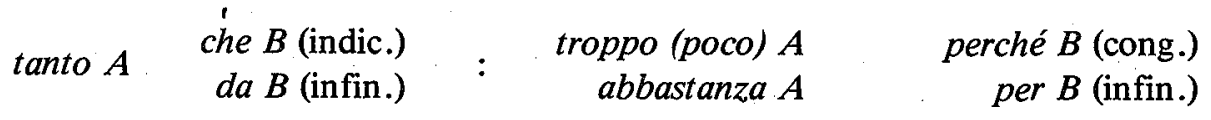

La struttura formale delle frasi di [ $\alpha$ adeguatezza] non è quella delle consecutive ma quella delle finali, appunto perché le frasi qui discusse riuniscono in sé tre componenti sintattico-semantiche:

$\begin{array}{lll}\text { la causa è/non presen- } & \text { necessario } & \text { produrre un de- } \\ \text { te ad un certo grado } & \text { per } & \text { terminato effetto } \\ \text { (comp. quantitativa) } & \text { (comp. finale) } & \text { (comp. consecutiva) }\end{array}$


Concludiamo che tanto il lato formale quanto quello semantico distinguono le frasi di [ $\alpha$ adeguatezza] dalle consecutive, per cui riteniamo giustificato interpretarle come un tipo di subordinate autonomo e coordinato alle altre.

7.6 Alla p. 478 si illustra la possibilità di trasformare le frasi subordinate implicite in esplicite e si dà l'esempio: Andrea è felice di essere stato promosso $\rightarrow$ Andrea è felice, perché è stato promosso. I due tipi di frasi ritornano nella tabella (p. 497), dove si legge anche l'esempio Sono felice di averti visto. Noi non crediamo che le frasi implicite ed esplicite siano in questo caso del tutto sinonime: infatti, mentre le esplicite (con perché) dicono effettivamente la causa, le implicite, anziché la causa, esprimono l'argomento dell'aggettivo e ne completano il significato. La differenza è ancor più sensibile con aggettivi come contento: sono contento di poter restare a casa e sono contento, perché posso restare a casa non sono affatto sinonimi (cfr. anche la differenza nella prosodia, cioè la pausa nella frase esplicita, espressa dalla virgola). A sostegno di quest'interpretazione crediamo di poter addurre due prove.

1) In periodi come Andrea è felice di essere stato promosso si può aggiungere un'altra subordinata (retta non dalla prima subordinata ma dalla reggente), di significato questa volta nettamente causale, e precisamente con la rematizzazione sia della causa che dell'effetto:

Andrea è felice di essere stato promosso, perché deve terminare lo studio quanto prima

Siccome Andrea deve terminare lo studio quanto prima, è felice di essere stato promosso.

Poiché in un periodo non sono possibili due subordinate omofunzionali rette dalla stessa reggente e non reciprocamente coordinate, e dato che le frasi aggiunte, introdotte da perché risp. siccome, sono indubbiamente causali, risulta che la implicita, introdotta da $d i$, non può essere causale.

2) Se sostituiamo il verbo in forma personale con un verbo impersonale (detto anche unipersonale), constatiamo che la struttura implicita $(d i+i n f$.) rimane possibile, quell'altra (perché + verbo pers.) invece no (oppure ha un altro senso):

\begin{tabular}{|c|c|c|}
\hline $\begin{array}{l}\text { Sono lieto di essere stato } \\
\text { promosso }\end{array}$ & $\Rightarrow$ & $\begin{array}{l}\text { Mi fa piacere di essere } \\
\text { stato promosso }\end{array}$ \\
\hline $\begin{array}{l}\text { Sono lieto, perché sono } \\
\text { stato promosso }\end{array}$ & & $\begin{array}{l}\text { ?*Mi fa piacere, perché sono }^{*} \text { stato promosso } \\
\text { sto }\end{array}$ \\
\hline
\end{tabular}

Anche questa differenza prova che i due tipi di frasi si distinguono. Si aggiunga, infine, che essi si distinguono anche in altre lingue (scr. sretan sam da (o sto) je moj sin položio ispit : sretan sam jer je moj sin položio ispit, ted. ich bin froh, daß 
mein Sohn die Prüfung bestanden hat : ich bin froh, weil mein Sohn die Prüfung bestanden hat ecc.).

7.7 Qualcosa di incomprensibile e insostenibile si legge alla p. 473: si afferma che certe congiunzioni [le ipotattiche], sia che si premettano ad una frase o che si collochino alla fine di essa, suscitano un senso di sospensione e provocano una domanda. Gli esempi sono:

\section{quando devo uscire di casa...}

prendo l'ombrello quando...

Salta agli occhi di chiunque conosca un pochino l'italiano (anzi, la grammatica in genere) che anche nel secondo esempio la congiunzione non è alla fine della frase (che è, nota bene, la reggente!) ma si trova normalmente all'inizio della frase (subordinata), solo che questa non è espressa. La differenza consiste dunque unicamente nell'ordine reciproco delle frasi, mentre la congiunzione è sempre in testa alla subordinata. Se così non fosse, dovrebbero essere possibili frasi agrammaticali (agrammaticalissime, sit venia elativo!) come queste:

* $\dot{E}$ cominciato a piovere sono uscito di casa non appena

*Resto a casa mi sento poco bene perché

*Il tempo è brutto poiché, non andiamo in gita ecc.

7.8 Infine, per terminare con la sintassi, osserviamo in fretta che alla p. 498 la frase per urlare che facesse è dichiarata implicita, mentre è evidentemente esplicita, essendo il verbo in forma esplicita [ = personale]. La frase è sinonima di per quanto urlasse, esplicita anche questa.

\section{Alcuni problemi della formazione delle parole}

8.1 Le nostre osservazioni si concentrano praticamente su un solo problema: l'interpretazione dei derivati tipo prudenza, insipienza, perseveranza ecc. Alla $\mathrm{p}$. 532 prudenza è uno dei due esempi per la formazione nominale deaggettivale con il suffisso -enza (l'altro è esperienza); alla p. 534 si dà la formula prudente + -enza $\rightarrow$ prudenza (!); alla p. 556, nell'esercizio num. 4 , si ripete la coppia prudente $\rightarrow$ prudenza e si chiede all'alunno (tra l'altro) di formare derivati mediante i suffissi -anza e -enza dagli aggettivi insipiente e perseverante. Che cosa si può obiettare?

1) Anzitutto, se la formula data alla p. 534 fosse corretta, il risultato dovrebbe essere *prudentenza, non prudenza. La formula è dunque sbagliata: o la base non è prudente o il suffisso non è -enza.

2) Se dovessimo isolare in prudenza il suffisso -enza, risulterebbe come base il segmento prud-, che in italiano si può collegare, semmai, col verbo prudere; e poi- 
ché derivati deverbali con -enza ci sono (cfr. partenza), prudenza dovrebbe avere su per giù lo stesso significato come prurito.

3) Naturalmente, il suffisso in prudenza non è -enza, e così pure in insipienza; e anche in peserveranza si può isolare il suffisso -anza soltanto se il nome è derivato dal verbo perseverare, non certamente se è deaggettivale, da perseverante.

4) In tutti i casi discussi il suffisso è solo -ia, aggiunto alle basi che già escono in -ant-/-ent -. Nel processo di derivazione si assibila la / $t$ / in /ts/ e si perde successivamente la semivocale:

$$
\text { prudent }+i a \Rightarrow \text { prudenz }+i a \Rightarrow \text { prudenza. }
$$

Si tratta dello stesso tipo di formazione come nella coppia forte $\rightarrow$ forza, tipo di cui nella nostra grammatica non si parla. La regola della perdita della semivocale, pur essendo frequentissima, non è generalizzata e ineccepibile, poiché da inerte abbiamo inerzia (non *inerza) e da infante si ha infanzia (in quanto la coppia è motivata). In queste eccezioni consiste la differenza tra questo gruppo e quello dei derivati dalle basi in /č/ (audace $\rightarrow$ audacia, efficace $\rightarrow$ efficacia ecc.), dove si ha lo stesso suffisso -ia ma la perdita della semivocale è ineccepibile, perché è determinata da fattori fonotattici (inesistenza della sequenza /čj/) ed è automatica.

5) L'errore nell'analisi dei derivati prudenza ecc. è dunque evidente, ma, come attenuante a favore dei nostri autori, non bisogna tacere che lo stesso errore si trova anche in altri manuali: Regula-Jernej 1975, p. 76 (e ci sono vari altri errori ancor più gravi, come ad es. il suffisso /inesistente!/ -orico in esempi come storico, allegorico, p. 78, o il suffisso -udine in incudine, p. 76), M. Fogarasi 1983, p. 137 (suffisso -anza in eleganza, -enza in imminenza), Zingarelli 1983 (esempio prudenza s. v. -enza).

8.2 Alla p. 543, tra gli esempi per il prefisso $s u b-, s u-$, so-, il quale indica inferiorità ('sotto'), è finito anche l'aggettivo surreale, dove il prefisso è di significato esattamente opposto (superiorità; 'al di sopra') e non ha niente a che fare con $s u b$;, perché risale (come il corrispondente francese sur-) al lat. SUPER.

Opere citate:

Crystal 1971: D. Crystal, Linguistics, Harmondsworth.

Deanović-Jernej 1980: M. Deanović-J. Jernej, Talijansko-hrvatski ili srpski rječnik (Vocabolario italiano-croato o serbo), Zagreb.

Devoto-Oli 1971: G. Devoto-G. C. Oli, Dizionario della lingua italiana, Firenze.

Feuillet 1983: J. Feuillet, Se débarrassera-t-on un jour des parties du discours?, «Bulletin de la Société de Linguistique de Paris» (BSLP) 78/1, pp. 23-51.

Feuillet 1987: J. Feuillet, L'organisation des trois points de vue, «BSLP» 82/1, pp. $1-41$. 
Fogarasi 1983: M. Fogarasi, Grammatica italiana del Novecento, 2. ed., Roma. Hall 1952-53: R. A. Hall jr., The Classification of ecco and Its Cognates, «Romance Philology» 6, pp. 278-280.

Pottier 1958: B. Pottier, Introduction à la philologie hispanique II: Morphosyntaxe espagnole, Bordeaux (policopiato).

Regula-Jernej 1975: M. Regula-J. Jernej, Grammatica italiana descrittiva su basi storiche e psicologiche, 2. ed., Bern.

Saussure 1965: F. de Saussure, Cours de linguistique générale, Paris.

Tekavčić 1968a: P. Tekavčić, Sur le superlatif italien et roman, «Studia Romanica et Anglica Zagrabiensia» 25-26, pp. 23-42.

Tekavčić 1968b: P. Tekavčić, O tzv. apsolutnom superlativu u modernom talijanskom jeziku [Sul cosiddetto superlativo assoluto nell'italiano moderno], «Živi jezici» IX/1-4, pp. 15-20.

Tekavčić 1980: P. Tekavčić, Grammatica storica dell'italiano (I Fonematica, II Morfosintassi, III Lessico), Bologna.

Tekavčić 1982: P. Tekavčić, Intorno ad alcuni «ribelli» nella tipologia, Osservazioni contrastive su materiale italiano, serbocroato e latino, «Studije iz kontrastivne analize italijanskog i srpskohrvatskog jezika» 2, Beograd, pp. 5-22.

Tesnière 1959: L. Tesnière, Eléments de syntaxe structurale, Paris.

Zingarelli 1983: N. Zingarelli, Vocabolario della lingua italiana, 11. ed. a cura di Miro Dogliotti e Luigi Rosiello, Bologna.

Sažetak

NEKA RAZMIŠLJANJA U POVODU JEDNE OD NAJNOVIJIH GRAMATIKA TALIJANSKOGA JEZIKA

U ovom se prilogu iznose neke kritičke primjedbe na veliku gramatiku talijanskog jezika La lingua e i testi autorâ P. Agazzi, A. Fallica i A. Menegoi (Bergamo 1988). Pored gramatičke materije u uobičajenom smislu taj udžbenik daje i temeljne pojmove opće lingvistike (jezični znak, funkcije jezika, značenje itd.), pragmatike, sociolingvistike, jezične komunikacije $i$ analize tekstova, a sadrži i pregled povijesti talijanskog jezika i elemente dijalektologije. Vrlo je moderno impostiran i liberalan u pogledu jezične pravilnosti, daleko od tradicionalnoga purizma. Ipak, unatoč svoj suvremenosti ima u toj gramatici i diskutabilnih, pa i pogrešnih tvrdnji, kao i posve tradicionalističkih postupaka. Ovdje se podvrgavaju kritici ova područja: 1) teorija jezika i opća lingvistika (pogrešna tvrdnja da su ljudi dogovorom nadjenuli riječima značenja); 2) morfematska analiza (prvenstveno glagolskih) oblika (određene nepotpunosti i/ili nedosljednosti); 3) status elativa (koji je i ovdje uključen u komparaciju a u nju ne spada) i elativ prilogâ kao jedini oblik superlativa prilogâ; 4) heterogenost klase prilogâ (među koje je ubrojena i riječ ecco); 5) pogrešna ili nedovoljna analiza nekih tipova rečenica; 6 ) potpuno pogrešna tvorbena analiza izvedenice prudenza (i nekih sličnih), kao da je izvedena sufiksom -enza. 